\title{
Integrated approach to e-learning enhanced both subjective and objective knowledge of aEEG in a neonatal intensive care unit
}

\author{
Woei Bing $\underline{\text { Poon }}^{1,2,3}$, MRCPCH, FAMs, Vina $\underline{\text { Tagamolila }}{ }^{1}$, MD, Ying Pin Anne $\underline{\text { Toh }}^{1}$, MBBS, Zai Ru $\underline{\text { Cheng }}{ }^{1}$, MRCPCH, MBBS
}

INTRODUCTION Various meta-analyses have shown that e-learning is as effective as traditional methods of continuing professional education. However, there are some disadvantages to e-learning, such as possible technical problems, the need for greater self-discipline, cost involved in developing programmes and limited direct interaction. Currently, most strategies for teaching amplitude-integrated electroencephalography (aEEG) in neonatal intensive care units (NICUs) worldwide depend on traditional teaching methods.

METHODS We implemented a programme that utilised an integrated approach to e-learning. The programme consisted of three sessions of supervised protected time e-learning in an NICU. The objective and subjective effectiveness of the approach was assessed through surveys administered to participants before and after the programme.

RESULTS A total of 37 NICU staff (32 nurses and 5 doctors) participated in the study. $93.1 \%$ of the participants appreciated the need to acquire knowledge of aEEG. We also saw a statistically significant improvement in the subjective knowledge score $(p=0.041)$ of the participants. The passing rates for identifying abnormal aEEG tracings (defined as $\geq 3$ correct answers out of 5 ) also showed a statistically significant improvement (from $13.6 \%$ to $81.8 \%, p<0.001$ ). Among the participants who completed the survey, $96.0 \%$ felt the teaching was well structured, $77.8 \%$ felt the duration was optimal, $80.0 \%$ felt that they had learnt how to systematically interpret aEEGs, and $70.4 \%$ felt that they could interpret normal aEEG with confidence.

CONCLUSION An integrated approach to e-learning can help improve subjective and objective knowledge of aEEG.

Keywords: adult learning, aEEG teaching, continuing professional education, e-learning, integrated approach to e-learning

\section{INTRODUCTION}

Information technology has dramatically changed the way healthcare staff teach, learn and work. With the introduction of newer technologies, coupled with the needs of the local Residency Programmes and the rollout of Academic Medicine Centres (AMCs) in Singapore, there is an increased need for effective and scalable methods of conducting continuing professional education.

E-learning is a new strategy that may enable teaching to become more effective and multisensory in nature. Meta-analyses have shown that Internet-based continuing medical education (CME) programmes are just as effective in imparting knowledge as traditional formats of CME. ${ }^{(1)}$ However, e-learning also has disadvantages such as the possibility of technical problems, the need for greater self-discipline, the cost of developing programmes, and the lack of direct contact between the learner and teacher. ${ }^{(2)}$

Amplitude-integrated electroencephalography (aEEG), a continuous and noninvasive bedside test, is used to monitor the electrical activity of the brain. It is increasingly used in neonatal intensive care units (NICUs) worldwide to screen for, diagnose, monitor and prognosticate various neurological conditions (e.g. seizures, hypoxic-ischaemic encephalopathy). This technological advancement creates a learning need due to the complex nature of aEEG patterns and the need for real-time interpretations with patient management implications. However, the teaching of aEEG interpretation is also highly variable in terms of methodology, quality and effectiveness. In most parts of the world, aEEG interpretation is taught using traditional methods comprising lectures and tutorials.

To address the possible shortcomings of e-learning, we instituted an integrated approach to e-learning for aEEG teaching. This was done by incorporating online modules with supervised protected time, which provided opportunity for interaction between the teacher and the learner at the end of each online session. The aim of the present study was to assess the effectiveness of this integrated approach to e-learning in the teaching of aEEG.

\section{METHODS}

The e-learning programme, consisting of three modules of online aEEG teaching lasting 1 hour each, was administered in the NICU of Singapore General Hospital for a period of three months, from September to December 2012. The modules covered: (a) the basics of aEEG; (b) the fundamentals of aEEG interpretation; and (c) recognition of seizures. Protected time was given for the online teaching sessions, which were followed by facilitated discussions, and questions and answers, supervised by any one of the four authors. For the NICU nurses, these protected times were arranged during shift overlap. The supervisor's role was to: (a) monitor the attendance rate of participants; (b) ensure smooth

${ }^{1}$ Department of Neonatal and Developmental Medicine, Singapore General Hospital, ${ }^{2}$ Duke-NUS Graduate Medical School, ${ }^{3}$ Yong Loo Lin School of Medicine, Singapore Correspondence: Dr Poon Woei Bing, Consultant Paediatrician and Neonatologist, Department of Neonatal and Developmental Medicine, Singapore General Hospital, 11 Third Hospital Ave, Singapore 168751. poon.woei.bing@sgh.com.sg 
technicalities with respect to playing of the videos of the online teaching; and (c) facilitate discussion and answer queries at the end of each session. The supervisor for a particular module also served as the teacher/tutor for that module. Due to the complexity involved in scheduling the NICU nurses who worked different shifts, the modules might not have been administered in sequence. The number of participants for each small group teaching ranged from three to ten.

Invigilated anonymous survey questionnaires were administered before and after the three-module programme. The order of the aEEG questions and answer options were scrambled for both survey sessions, and all question papers were collected at the end of the sessions. No discussion regarding the questionnaire was allowed and the answers were not released to the participants. The written survey consisted of 25 questions (see Appendix), out of which five were objective questions testing the participant's ability to correctly identify aEEG tracings. These five tracings were chosen from among six possible aEEG pattern traces (i.e. continuous normal voltage, discontinuous normal voltage, continuous low voltage, burst suppression, inactive and seizure activity).

Statistical analysis was performed using the Statistical Package for the Social Sciences version 17 (SPSS Inc, Chicago, IL, USA). Continuous variables were expressed as median and range. Statistical significance for categorical data was determined by using Chi-square test, and paired Student's $t$-test was used to compare pre- and post-programme survey results. The present study was approved by the Institution Review Board.

\section{RESULTS}

A total of $37 \mathrm{NICU}$ staff (32 nurses and 5 doctors) were involved in the present study. The demographics of the participants are summarised in Table I. Among the 37 participants, 93.1\% responded that they appreciated the need to acquire knowledge of aEEG.

Based on the survey conducted at the completion of the programme, $77.8 \%$ (21/27) of the participants felt that the duration of the teaching was optimal, $96.2 \%(25 / 26)$ felt that the teaching was well structured, $80.0 \%(20 / 25)$ felt that they had learnt a systematic way of interpreting aEEGs, and 70.4\% (19/27) felt that they could interpret normal aEEGs with confidence. When the pre- and post-programme survey results were compared, we found a statistically significant improvement in the participants' subjective knowledge score (subjectively ranked between 1 to 10), with median improvement from pre- to post-programme score of 1 (range: -1 to $+2 ; p=0.041$ ). However, more objectively, the passing rates for identifying abnormal aEEG tracings (defined as $\geq 3$ correct answers out of 5) also showed a statistically significant improvement (from $13.6 \%$ to $81.8 \%, p<0.001$ ).

\section{DISCUSSION}

E-learning offers many advantages, including ease of access, flexibility of learning, continual availability of course content, and reduction in the cost and time expended by the students. E-learning also enables students to personalise the content and define the pace of learning, as well as fosters self-study and self-
Table I. Demographics of the study participants $(n=37)$.

\begin{tabular}{|c|c|}
\hline Demographic & No. (\%) \\
\hline \multicolumn{2}{|l|}{ Position } \\
\hline Staff Nurse & $29(78.4)$ \\
\hline Nurse Clinician/Nursing Manager & $3(8.1)$ \\
\hline Resident/Senior Resident/Registrar & $4(10.8)$ \\
\hline Consultant/Senior Consultant & $1(2.7)$ \\
\hline \multicolumn{2}{|l|}{ Age group (yr) } \\
\hline $20-25$ & $10(27.0)$ \\
\hline $26-30$ & $9(24.3)$ \\
\hline $31-35$ & $4(10.8)$ \\
\hline $36-40$ & $0(0)$ \\
\hline $41-45$ & $3(8.1)$ \\
\hline$\geq 46$ & $3(8.1)$ \\
\hline No response & $8(21.6)$ \\
\hline \multicolumn{2}{|l|}{ Gender } \\
\hline Female & $35(94.6)$ \\
\hline Male & $1(2.7)$ \\
\hline No response & $1(2.7)$ \\
\hline \multicolumn{2}{|l|}{ Years of working in neonatology } \\
\hline$<1$ & $1(2.7)$ \\
\hline $1-5$ & $21(56.8)$ \\
\hline $6-10$ & $3(8.1)$ \\
\hline$>10$ & $9(24.3)$ \\
\hline No response & $3(8.1)$ \\
\hline \multicolumn{2}{|l|}{ Experience with aEEG } \\
\hline Yes & $14(37.8)$ \\
\hline No & $16(43.2)$ \\
\hline No response & $7(18.9)$ \\
\hline \multicolumn{2}{|l|}{ Educational level } \\
\hline Diploma & $15(40.5)$ \\
\hline Degree & $12(32.4)$ \\
\hline Postgraduate & $3(8.1)$ \\
\hline No response & 7 (18.9) \\
\hline
\end{tabular}

learning capabilities among students. Moreover, using e-learning platforms, it is possible to train a large number of people at the same time and reach audiences that are geographically dispersed. Among the many benefits of e-learning, learning delivery is the most often cited advantage over traditional methods. Learning delivery includes greater accessibility to information, ease of updating and distribution of content, content standardisation, and accountability. ${ }^{(3)}$

In medical teaching, e-learning facilitates a revolutionary shift toward contemporary adult learning theory, ${ }^{(3)}$ in which educators no longer serve as merely distributors of content, but facilitators of learning and assessors of competency. In this new paradigm, adults learn by: (a) relating new learning to past experiences; (b) linking learning to specific needs; and (c) practical application of the learning. Thus, e-learning results in more effective and efficient learning experiences. The context to which e-learning is applied may be important. Among the participants in the present study, 93.1\% acknowledged their need to learn about aEEG and $37.8 \%$ had seen aEEG being used on patients (and therefore had some related experience in the use of aEEG). A previous study found that e-learning is most effective when healthcare professionals are allowed to learn at their own time and to select topics that are most relevant to their professional practice, accommodating just-in-time learning. ${ }^{(4)}$ 
However, e-learning also has its disadvantages. It is associated with the possibility of technical problems, the need for greater self-discipline for self-directed learning, added costs of developing programmes, and the lack of direct contact between learners and teachers. Cook et al conducted a systematic review that looked at ways to improve Internet-based learning, and concluded that interactivity, practice exercises, repetition and feedback were associated with improved learning outcomes; however, there were inconsistencies across the studies reviewed, which may have tempered the conclusions of the review. ${ }^{(5)}$

As part of our strategy to improve e-learning, we implemented an integrated approach that aimed to address the associated drawbacks of e-learning. This approach involved supervised protected time for e-learning. In this way, any possible technical problems can be avoided, as the supervisor was present to ensure that the online teaching and video modules were played, and any issue with a lack of self-discipline could also be circumvented. In addition, supervised protected time allowed for direct interaction between the learner and the teacher after each e-learning session; this addresses the main disadvantage of e-learning, i.e. the lack of direct contact between learners and teachers and the inability to facilitate discussions. The method of information delivery used in the present study, which 'blends' face-to-face learning with e-learning, is currently the trend in e-learning. ${ }^{(6)}$ Other strategies can be used to enhance learnerteacher interaction, including the use of electronic mails and/or real-time feedback channels (e.g. online forums, bulletin boards, Listserv, newsgroups and weblogs). Also, e-learning programme software can be used to track participation, thereby improving participation rates. ${ }^{(7)}$

While e-learning is unable to duplicate some aspects of training that takes place in a classroom (e.g. demonstration of clinical procedures, actual practice with machines, and learning via shared experiences), ${ }^{(7)}$ it has been shown to be as effective as expert-led learning of practical procedures such as urinary catheterisation. ${ }^{(8)}$ This improved skill transfer is possibly due to humans' limited attentional capacity for motor learning and the fact that novice learning can be impaired in the presence of too much information from experts. ${ }^{(8)}$ Other criticisms of e-learning include greater delay in the creation and preparation of online courses compared with traditional methods of learning, as well as higher costs incurred in the implementation of e-learning programmes. However, this may not necessarily be true in every context, particularly if there is a need for multiple sessions and/or when multiple geographical locations are involved. In addition, e-learning confers the ability to modify lesson contents more quickly, and if harnessed properly for adult learning, it may result in greater effectiveness than traditional methods; these attributes may even make e-learning more cost effective than traditional methods. ${ }^{(9)}$

E-learning comprises not only hardware and software components, but also pedagogy and content. In fact, the success of any e-learning programme is very much dependent on the latter two components (i.e. pedagogy and content). In order to adapt a subject for e-learning, there needs to be careful consideration of the context to which e-learning is to be applied, and existing educational and learning models may need to be redefined during this adaptation process. Cooperative learning and tutoring are two key concepts that should be incorporated to ensure success. ${ }^{(10)}$ Tutoring in an e-learning environment involves acting in the role of facilitator, instructor or moderator. Tutor-facilitators are methodology experts who facilitate the learning process and sustain the student's motivation. Tutor-instructors are usually experts on the subject, and their role includes addressing the questions of students. Tutor-moderators define the interactions, mainly by moderating the discussions that occur in the learning process.

In our opinion, the results of the present study serve to vindicate the effectiveness of an integrated and holistic approach to e-learning. The approach used incorporated both Internetbased learning and face-to-face interactions, thus enhancing the strengths of e-learning while addressing its weaknesses. We believe that this approach is worthy of consideration for various settings of continuing professional education - from residency programmes and advanced specialist trainings to nursing education. It was particularly noteworthy that the NICU nurses were satisfied with the programme, as e-learning gave them the opportunity to attend such teaching sessions, which they would otherwise miss due to the hospital's shift system.

The present study was, however, not without limitations. As this was a cross-sectional study, we did not address knowledge retention over time, or the ability of its participants to convert the theoretical knowledge gained into practical knowledge that could be applied at the bedside. We were also unable to compare e-learning alone with an integrated e-learning approach, as our participants had, prior to the start of the study, requested that assistance and question-and-answer sessions be included in the programme; hence, no e-learning only programme was conducted. In addition, the setting and training needs were specific to NICU healthcare professionals, who were already well-motivated individuals. However, this limitation would apply to many adult learning approaches, in which the adult learners are interested to acquire knowledge that they can immediately apply and/or information that relates to their past experiences. Another limitation pertains to the demographics of our study cohort, which consisted mainly of nurses (as opposed to doctors) who were young learners (aged $\leq 30$ years). A large proportion of our participants had either a diploma or degree and $\leq 5$ years of work experience in the specialty. Therefore, there is a possibility that these younger, well-educated learners were more open to e-learning. Finally, the present study was also limited due to the variable response rates for each question in the survey, with the non-response rate as high as $32 \%$ for some questions.

Future research in e-learning falls into either macro views of the context for e-learning such as systems, organisations and cultures, or micro perspectives that are concerned with individual learners, interventions and technologies. ${ }^{(11)}$ E-learning research in general covers a wide range of issues and perspectives, including the context for e-learning, theoretical perspectives (both educational and cultural), policy and politics, and technical 
and implementation designs. ${ }^{(5)}$ Evidence to guide decisions on research should be derived from studies (e.g. qualitative analyses) that are designed to clarify the relationships between potential advantages of e-learning and specific topics, course objectives and learner characteristics. ${ }^{(5)}$

In conclusion, e-learning ensures that teaching is labour efficient and of a consistently high quality. It also enhances learners' autonomy, as it allows them access to learning materials at their own convenience and pace. In order to reap the most benefits out of e-learning, we should consider employing an integrated approach that involves the use of both information technology and interaction/feedback, such as face-to-face time. E-learning should also be applied in appropriate situations, and should incorporate carefully considered pedagogy that takes into account adult-learning theories.

\section{ACKNOWLEDGEMENT}

The e-learning materials used in the present study are copyrighted to aEEG Coach (C) 2012, gRN NICU Inc.

\section{REFERENCES}

1. Wutoh R, Boren SA, Balas EA. eLearning: a review of Internet-based continuing medical education. J Contin Educ Health Prof 2004; 24:20-30.

2. Mariani AW, Terra RM, Pêgo-Fernandes PM. E-learning: from useful to indispensable tool. Sao Paulo Med J 2012; 130:357-9.

3. Ruiz JG, Mintzer MJ, Leipzig RM. The impact of E-Learning in medical education. Acad Med 2006; 81:207-12.

4. Pullen DL. An evaluative case study of online learning for healthcare professionals. J Contin Educ Nurs 2006; 37:225-32.

5. Cook DA, Levinson AJ, Garside $\mathrm{S}$, et al. Instructional design variations in internet-based learning for health professions education: a systematic review and meta-analysis. Acad Med 2010; 85:909-22.

6. Farrell M. Learning differently: e-learning in nurse education. Nurs Manag (Harrow) 2006; 13:14-7.

7. Nelson EA. E-learning. A practical solution for training and tracking in patient-care settings. Nurs Adm Q 2003; 27:29-32.

8. Walsh CM, Rose DN, Dubrowski A, et al. Learning in the simulated setting: a comparison of expert-, peer-, and computer-assisted learning. Acad Med 2011; 86(10 Suppl):S12-6.

9. Lain D, Aston J. Literature review of evidence on e-learning in the workplace. Institute for Employment Studies 2005: 21-22.

10. Sajeva M. E-learning: Web-based education. Curr Opin Anaesthesiol 2006; 19:645-9.

11. Masters K, Ellaway R. e-Learning in medical education Guide 32 Part 2: Technology, management and design. Med Teach 2008; 30:474-89. 


\section{APPENDIX}

Thank you for participating in this survey, which aims to assess the effectiveness of online amplitude-integrated electroencephalography (aEEG) teaching. It will take five minutes to complete this survey. Please circle/fill in the appropriate answer.

1. I have / have not started on the aEEG video teaching.

If you have started on the teaching, please indicate the number of lessons you have attended: $1 / 2 / 3 / 4 / 5 / 6$

Specify which lessons you have attended (you may circle more than one number): 1/2/3/4/5/6

2. I am a
a. staff nurse
b. enrolled nurse
c. nurse manager
d. resident doctor/medical officer
e. clinical associate
f. registrar/associate consultant
g. consultant/senior consultant

3. My age is years old.

4. I am male/female.

5. What is the highest level of education you have attained?
a. Diploma
b. Degree
c. Postgraduate
d. Others:

6. Number of years working in neonatalogy: years

7. I have/have not worked with aEEG monitoring before.

If you have, please specify where: ; and how long: months/years

8. On a scale of $1-10$, with 1 being poorest and 10 being very knowledgeable, my current level of knowledge of aEEG is

9. The aEEG machine in our unit is a
a. single channel aEEG
b. dual channel aEEG
c. same as the EEG machine in the EEG lab
d. no idea

10. I feel that aEEG is a useful tool for monitoring patients: Yes/No

11. I feel that aEEG makes a difference in patient management: Yes/No

12. I feel that it is necessary for me to know aEEG in detail: Yes/No

13. Based on my knowledge, the following are the indications for aEEG (you may circle multiple answers):
a. Unsure
b. Hypoxic-ischaemic encephalopathy (HIE) patients on cooling
c. Intraventricular haemorrhage (IVH)
d. Congenital brain abnormalities
e. Kernicterus
f. Suspected seizures
g. Routine monitoring for all sick newborn 


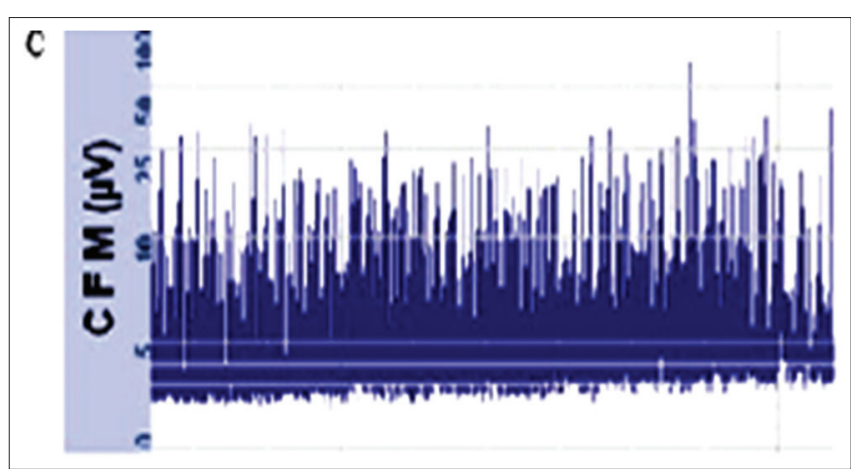

14. The above aEEG trace shows
a. flat trace (FT)/inactive
b. continuous normal voltage (CNV)
c. low voltage (LV)
d. burst suppression (BS)
e. discontinuous normal voltage (DNV)
f. seizure (s)

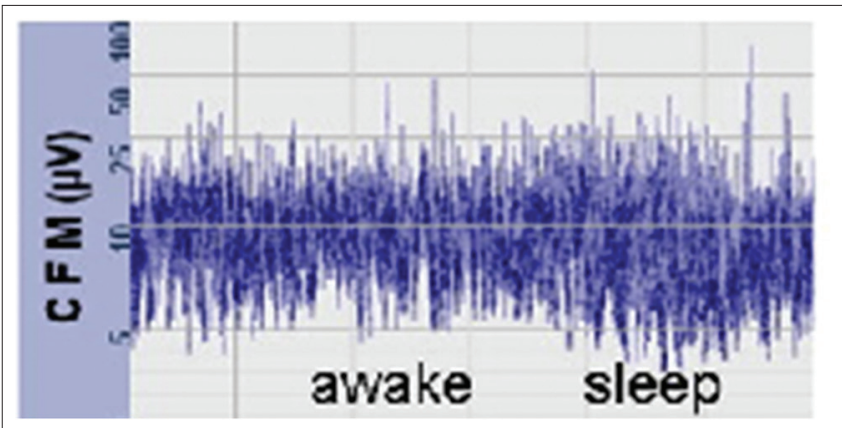

15. The above aEEG trace shows
a. flat trace (FT)/inactive
b. continuous normal voltage (CNV)
c. low voltage (LV)
d. burst suppression (BS)
e. discontinuous normal voltage (DNV)
f. seizure(s)

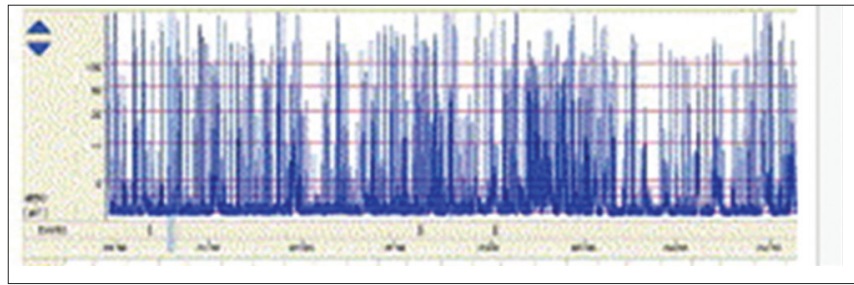

16. The above aEEG trace shows
a. flat trace (FT)/inactive
b. continuous normal voltage (CNV)
c. low voltage (LV)
d. burst suppression (BS
e. discontinuous normal voltage (DNV)
f. seizure $(s)$

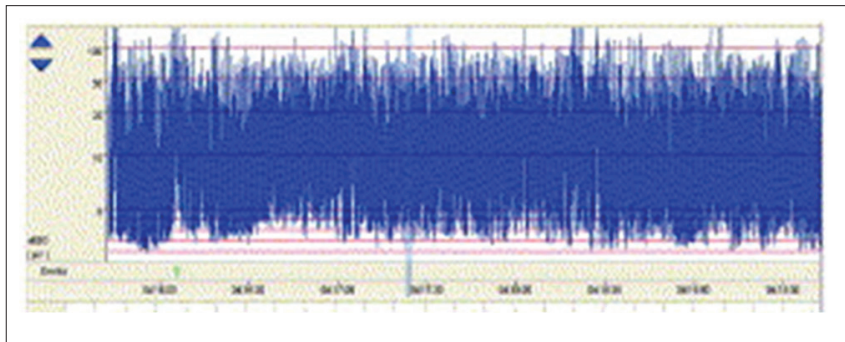

17. The above aEEG trace shows
a. flat trace (FT)/inactive
b. continuous normal voltage (CNV)
c. low voltage (LV)
d. burst suppression (BS)
e. discontinuous normal voltage (DNV)
f. $\quad$ seizure(s) 


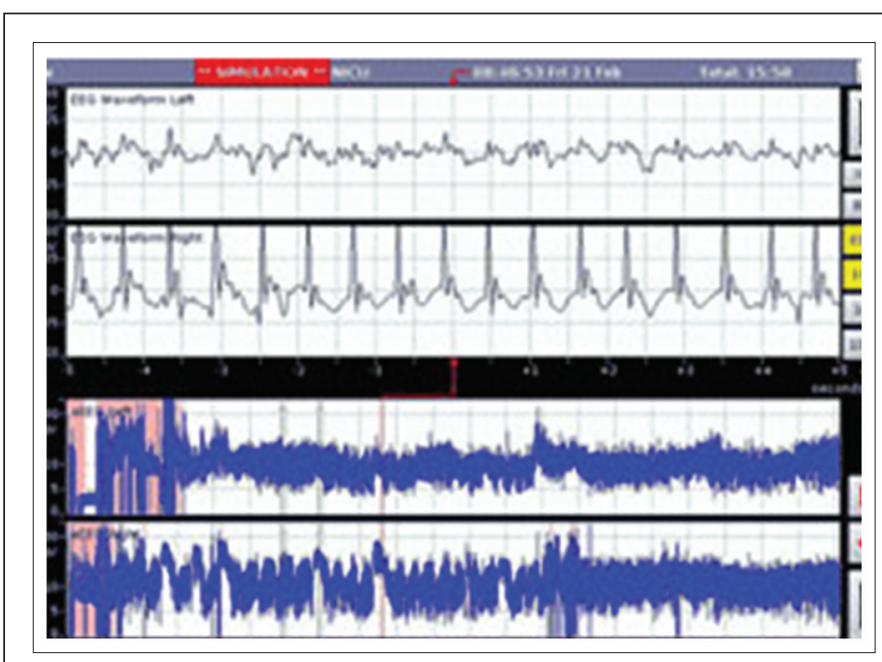

18. The above aEEG trace shows
a. flat trace $(\mathrm{FT}) /$ inactive
b. continuous normal voltage (CNV)
c. low voltage (LV)
d. burst suppression (BS)
e. discontinuous normal voltage (DNV)
f. seizure(s)

If you have yet to attend the aEEG online teaching, please answer the following questions:

19. My current teaching for aEEG is structured: Yes/No/Never trained before

20. I now have a systematic way to interpret aEEG: Yes/No

If you have attended the aEEG online teaching, please answer the following questions:

21. I felt that the structured aEEG teaching was useful: Yes/No

22. The teaching duration was: too short/just right/too long.

23. The teaching was: easy to understand/not easy to understand.

24. I now have a systematic way to interpret aEEG: Yes/No

25. I can identify a normal aEEG: Yes/No 